\title{
Tympanometric screening for Otitis media of paediatric patients with respiratory tract infection in rural setting a prospective observational study
}

\author{
Rajamani S.K. ${ }^{1}$, Choudhary V.C. ${ }^{2}$, Mogre D.A. ${ }^{3}$ \\ ${ }^{1}$ Dr. Santhosh Kumar Rajamani, Associate Professor of Otorhinolaryngology, Head and Neck Surgery, Department of \\ E.N.T, ${ }^{2}$ Dr. Vinod C. Choudhary, Associate Professor of Paediatrics, ${ }^{3}$ Dr. Dilesh A Mogre, Senior Resident, Department \\ of E.N.T, all authors are affiliated with B.K.L Walawalkar Rural Medical College, Chiplun, Ratnagiri district of \\ Maharashtra, India; Maharashtra University of Health Sciences, Nasik, Pune, Maharashtra India.
}

Corresponding Author: Dr. Santhosh Kumar Rajamani, Associate Professor of Otorhinolaryngology, Head and Neck Surgery, B.K.L Walawalkar Rural Medical College, Chiplun, Ratnagiri District of Maharashtra, Address: C-603 Redwoods CHS C wing Vasanth Gardens Mulund West Mumbai (Bombay) India. E-mail: minerva.santh@gmail.com

\begin{abstract}
Introduction: Early identification of hearing impairment in childhood is imperative, as even a mild hearing loss can have long term consequence on the development of the Central Nervous System. Many children develop transient, fluctuant deafness due to Middle ear effusion, especially during episodes of Common cold. In this research we try to develop a Screening protocol using Impedance Audiometry for early identification of Middle ear effusions. Materials and Methods: Children between 7 months to 6 years of ages, with no previous history of hearing impairment or ear disease, who were suffering from Upper respiratory tract infection (Common cold) were selected as targets of screening. These children were then subjected to a Screening Tympanometry. A simple, quick and accurate method of screening for Middle ear fluid was "Peak" or "No peak" approach was employed to judge the curves. If a curve was obtained (similar to Jerger's classification-type "A") child was deemed to have "Pass". A type "B" curve was deemed highly positive and was labelled "Fail+" and any other trace like type "C" or just reduced type "A" or "As" labelled just "Fail". Otoscopic and endoscopic examination and diligent search was carried out for signs of Middle ear effusion and confirmation was done. This was cross checked by 2 authors (First and third author) and findings confirmed. Results: Dervan child screening protocol is $91.67 \%(92 \%)$ sensitive and 94.23 (94\%) specific is detection of Middle ear effusions. Conclusion: Dervan child Middle ear effusion protocol can be used in a cost efficient, scalable and sustainable method of screening children for Middle ear effusion. Tympanometry in selected high risk population is an accurate and reliable test for detection of Middle ear effusion
\end{abstract}

Keywords: Middle Ear Effusion, Serous Otitis Media, Secretory Otitis Media, Impedance Audiometry, Tympanometry, Electroacoustic Impedance Tests, Acoustic Impedance Tests

\section{Introduction}

Early Identification of Middle Ear Disease in Children is important because Hearing impairment even mild to moderate levels can lead to irreversible consequence on the development of the Nervous system. Despite large scale use of Otoacoustic Emissions for screening of Newborns and Infants, most children will develop

\begin{abstract}
transient Hearing impairment which is present only during episodes of common cold (Upper Respiratory Tract Infection URTI). Many children who develop episodic Middle ear effusion will suffer from variety of fluctuant deafness for weeks and months, which can impact the development of the Nervous system [1].
\end{abstract}

\section{Materials and Methods}

Tympanometric Screening is a strategy to detect Children who have a middle ear effusion from those who do not in a pain-free, safe, scalable, quick, and cost-effective manner. The main objective of this Tympanometric screening is to

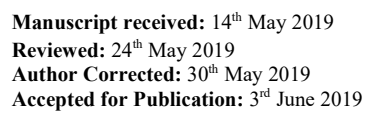


minimize the consequences of hearing impairment or chronic middle ear disease as early as possible so that the disorder will not interfere with the Neuro-development of the child and hence lead to a disabling condition. In 1977, a special task force constituted by the American Speech-Language-Hearing Association (referred to as the ASHA) studied the use of Impedance measures in screening for middle ear disease and concluded that there was a value of Tympanometric screening. The task force recommended screening of special higher risk groups of children, namely Native American children, those with sensorineural hearing loss, children with development delay and children with Down's syndrome, cleft palate and other craniofacial anomalies [2].

The percentage of cases correctly diagnosed by the test is called its Sensitivity, and the percentage of negative results from the tests in normal subjects is called the Specificity of the test. This ASHA method of Impedance screening suffers from low specificity, where in a large number of normal children also get referred for further evaluation [3].

\section{Historical Tympanometric screening programs}

ASHA guideline (1997) for screening children from 7 months of age to 6 years uses the following criterion [4]

Table-1: ASHA guideline (1997) for screening children from 7 months of age to 6 years.

\begin{tabular}{|c|l|}
\hline Serial & \\
\hline 1 & Case history \\
\hline 2 & Examination of Ear namely Ear canal and Tympanic membrane \\
\hline 3 & Impedance Audiogram with 220 or $226 \mathrm{~Hz}$ Probe tone \\
\hline
\end{tabular}

Hirtshal program for Tympanometric screening of children uses only tympanometry and no acoustic reflex measurement. A normal tympanogram (type "A") is considered pass. The remaining children receive a second measurement in 4 to 6 weeks to allow resolution of effusion and all cases with flat (type "B") tympanograms are referred. Children still remaining from second testing receive a third test 4 to 6 weeks later. Children with normal Type "A" tympanograms or tympanograms having peaks in the range of 100 to $200 \mathrm{da}$ Pa are excluded. Those having flat (Type "B") tympanograms or tympanograms with peaks below $200 \mathrm{daPa}$ at the third test are referred ( 8 to 12 weeks after initial testing). Hirtshal screening protocol has, sensitivity of $80 \%$ and specificity of $95 \%$ and a referral rate of 8 to $10 \%$ only [5].

Type of Study, sampling and ethical considerations- This was a unicentric, hospital based setting, cross-sectional, epidemiological, population based research and the accepted level of significance $\mathrm{p}$ value of 0.05 ( $95 \%$ significance). The sampling was random paediatric patients who attended the clinic for symptoms of upper respiratory tract infection. Data was collected by the first author with cross verification done by the other authors.

There were no ethical issues involved as the test was conducted free of cost for the patients and Tympanometry is non invasive, rapid, painless and objective. Staging system of Pars tensa retraction suggested by Sade was used to clinically grade the degree of retraction. Neither treatment nor surgical intervention was included in this study.

\section{Development of our Screening protocol- Dervan child hearing screening protocol [4]}

\section{Inclusion and exclusion criteria}

In neonates the standard $226-\mathrm{Hz}$ tympanograms donot provide correct diagnostic information due to immaturity of the Ossicles, horizontal orientation of the Tympanic membranes [6]. In addition the Tympanogram of Neonates is claimed to be of a notched "M" shape [7]. Neonates were thus excluded from the study.

For selection of the screening the following inclusion criteria were used.

1. Children between 7 months to 6 years of ages.

2. Any hearing loss beyond $55 \mathrm{db}$ was deemed not due to Serous Otitis media and was excluded from this protocol. Children with any evidence ear discharge were excluded from the study as these would be cases of Chronic Otitis Media (COM / CSOM) not a subject of this current research. 
3. Those children suffering from Upper respiratory tract infection, Common cold, with a running nose, low grade fever, minimal cough and fatigue. Children generally develop Middle ear effusion only under the conditions of an upper respiratory tract infection or common cold. Most of these effusions go undetected and under diagnosed.

4. External ear canal was examined under light to rule out Cerumen or Ear canal atresia or any other ear canal pathology which could lead to erroneous interpretation of our Tympanometric findings. Smaller ear canals and horizontal placement of Tympanic membranes, lack of cooperation, and operator variability of findings (subjectivity) are few problems associated with Otoscopic examination. Note Otoscopic examination of ear drum was not done as the first step as the protocol was for screening purpose.

Peak or No peak Impedance Audiogram- Tympanometry equipment was calibrated on daily basis. Mother was told to hold the child on her lap while an Impedance audiogram was recorded with a hand held probe. A simple, quick and accurate method of screening for Middle ear fluid was "Peak" or "No peak" approach. Here the screening test was deemed "Pass" if a peak was found in the Impedance graph while test was deemed "fail" if no peak was observed.

A type "B" trace was deemed highly positive and labelled as "Fail+" and any other trace like type "C" or just reduced type "A" or "As" was labelled as "Fail" [8].Multi-component tympanometry (MFT) tympanometry and non standard frequencies (other than standard 226-Hz) were not used as these are more experimental and hence not standardized [9].

Otoscopic examination and scoring system of Middle ear retraction- This was carried out for all children who "failed" the "Peak or No peak" test. Diligent examination of the Tympanic membrane was done to look for presence of fluid or retraction. The following staging system of Pars tensa retraction suggested by Sade was used [10].

Stage I- retraction of tympanic membrane due to negative pressure in the middle ear. Anterior and posterior malleal folds are prominent and there is distortion of light reflex. Long process of incus is not visible

Stage II- retraction of tympanic membrane which is reaching the long process of Incus

Stage III- Middle ear atelectasis/collapse: Tympanic membrane touching the promontory and the ossicles but not adherent to these structures. The middle ear remains relatively intact.

The tympanic membrane is mobile though is restricted.

Stage IV- adhesive otitis media: Middle ear space is obliterated. Tympanic membrane is adherent to ossicles and promontory. No movements can be elicited.

This was marked as Sade 1, 2, 3, $\mathbf{4}$ on the case papers in red ink.

Pure tone Audiogram- This was carried out in a selected group of children whose ear was found pathological on Otoscopic screening. This was possible only if the child was cooperative, which was occasionally possible after conditioning.

The whole screening process takes under 50 seconds of time per child. Most patients who "pass" the screening impedance do not need any further evaluation. Those with "fail" and "fail+" were further evaluated.

Follow up screening was done after 4 to 6 weeks to allow spontaneous resolution of effusion. If the child still "Failed" the screening, this was taken up for necessary medical or surgical intervention.

Biostatistical analysis- Bio-statistical analysis was done using the Open source P.A.S.T Statistical software package [11]. A Confidence interval of $95 \%(p=0.05)$ was setup for the entire hypothesis tested in this study.

Analysis of variance test (ANOVA), Pearson's Chi-square test and linear regression modelling were done to compare the observed traits. Homogeneity of demographic and clinical data was thus ascertained. A nonparametric test to compare two samples such as the Mann-Whitney was used when Normality of our data was questionable [12]. 
Stage I ( $\mathrm{n}=133$ cases, 33 rejected due to criteria $1-4)$

1. Children between 7 months to 6 years (ASHA guideline 1997) [4]

2. Common cold (ideal time for effusions to appear)

3. No previous hearing or ear problem (previous pathology)

4. No obvious ear pathology seen with a pen-torch light (affecting the test)

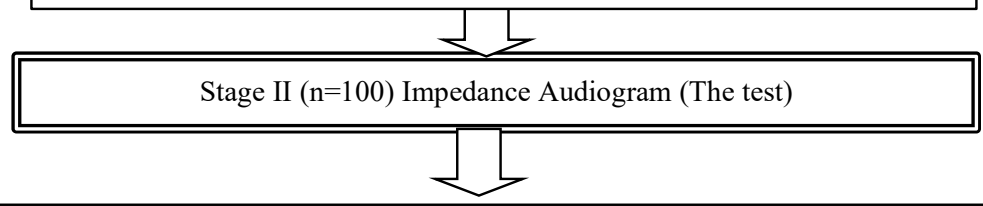

Is apeaked curve immediately visible on monitor? Similar to Jerger Type "A" Uncrossedipsilateral Acoustic reflex present (+) or absent (-)

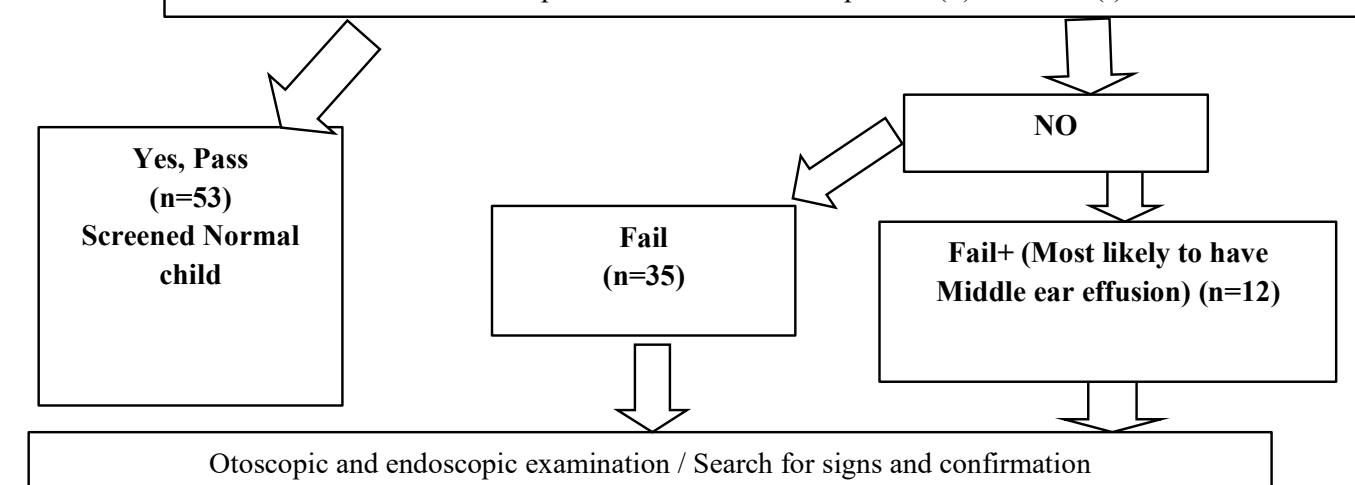

Otoscopic and endoscopic examination / Search for signs and confirmation

$\underline{\text { Signs of Middle ear effusion }}$

- Bluish hue of the Tympanic membrane

- Actual fluid

- Hair line sign

- Meniscus sign

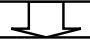

- Air bubbles

- Reddish colour of the Tympanic membrane

- Cartwheel sign

- Pars tensa retraction stage suggested by Sade
- Pure Tone Audiogram (if possible)

- Repeat after 4 to 6 weeks to allow resolution of effusion

- Refer for Surgical or Medical intervention if unresolved

\section{Fig-1: Dervan child screening protocol for detection of Middle ear effusion and transient deafness}

\section{Result}

Table I depicts demographic characteristics of participants in this Screening protocol. Children of from 7 months of age to 6 years, who attended the Paediatric medical clinic, for symptoms of common cold (which is the ideal time for effusions to manifest), having normal hearing child (No previous ear pathology) and no obvious ear canal pathology observed with a common pen-torch light (affecting the test) were inducted into the first stage of Screening.

A total 133 children were examined at Paediatric clinic, 33 were rejected in stage 1 and100 children were included for the study stage 2

The average age was 4.5 years (Standard deviation of 0.8 years), and the majority of suffering patients were female $(68 \%)$. There were no observed statistically significant differences in age, sex across disease types. 
Table-2: Baseline demographic characteristics of children who were screened.

\begin{tabular}{|c|c|}
\hline \multicolumn{2}{|c|}{ Screening participants demographics } \\
\hline Characteristic & No. $(\%)$ \\
\hline Female sex & $73 \%$ (73 females +27 males $)$ \\
\hline Mean (SD) age (y) & $4.5+/-0.8$ years \\
\hline \multicolumn{2}{|c|}{ Age (y) } \\
\hline 7 months- 1 year & $12(12 \%)$ \\
\hline $1-2$ & $16(16 \%)$ \\
\hline $2-3$ & $20(20 \%)$ \\
\hline $3-4$ & $11(11 \%)$ \\
\hline $4-5$ & $15(15 \%)$ \\
\hline $5-6$ & $12(12 \%)$ \\
\hline $6-7$ & $14(14 \%)$ \\
\hline \multicolumn{2}{|c|}{ Symptoms } \\
\hline Cough & $56(56 \%)$ \\
\hline Common cold, Running nose, fever & $100(100 \%)$ \\
\hline Ear block, Ear pain, holding the ear & $54(54 \%)$ \\
\hline \multicolumn{2}{|l|}{ Other co-morbidities, infections } \\
\hline Malnutrition (Skin fold thickness) & $34(34 \%)$ \\
\hline Acute tonsillitis & $27(27 \%)$ \\
\hline Cleft palate & $2(2 \%)$ \\
\hline
\end{tabular}

Table-3: Basic Tympanometric data of children who were screened.

\begin{tabular}{|c|c|c|}
\hline Tympanometric parameter & Mean quantitative values which were observed & Range +/- 2SD (SE) \\
\hline Ear canal Volume & $0.876 \mathrm{ml}$ & $0.42 \mathrm{ml}$ \\
\hline Compliance & $0.31 \mathrm{ml}$ & $0.27 \mathrm{ml}$ \\
\hline Pressure & $17 \mathrm{daPa}$ & $11 \mathrm{daPa}$ \\
\hline Gradient & $161 \mathrm{daPa}$ & $40 \mathrm{daPa}$ \\
\hline
\end{tabular}

A total of 133 cases were chosen, from which 33 were rejected by the paediatrician on the basis of inclusion criteria. These were as follows: 1. Children between 7 months to 6 years (ASHA guideline 1997), 2. Suffering from cold which is ideal time for effusions appear, 3. No previous hearing or ear problem and 4. No obvious ear pathology seen with a pentorch. Hundred children were included in this screening program, amongst which 55 children had an immediately visible peakon Impedance audiometer (Jerger Type "A"). These children were deemed "Pass" in the screening.

Those who did not have a visible reading on Tympanometry were deemed failed in the screening and were most likely suffering from permanent or transient middle ear pathology.A type "B" trace was deemed highly positive and labelled as "Fail+" and any other trace like type "C" or just reduced type "A" or "As" was labelled as "Fail". "Fail" category those with a "Fail" were examined with Otoscope and diagnostic endoscope.

Diligent search was done for signs of Middle ear effusion (illustrated in the chart) like meniscus sign, hair line sign and air bubbles in middle ear. Any positive finding was confirmed by a consensus between two surgeons (Principal author and Co-author). Thus Screening Impedance audiogram was compared with a gold standard test of direct visualization of the Middle ear effusion. 


\section{Discussion}

Aetiology and patho-physiology of paediatric middle ear diseases and conductive deafness- There is a phase differential between sound waves travelling in the air column and fluid filled cochlea which is site of perception of sounds [13]. This phase differential is overcome by the transformer action of the middle ear, tympanic membrane and ossicles [14]. Thus acting as an amplifier of sound and transmit it to the inner-ear fluid. If this conduction pathway is obstructed sound can still travel via the skin and through the bones of the skull and directly stimulate the cochlea [15]. This occurs at the cost of significant energy loss. The most common causes of conductive hearing loss include impacted wax in the external canal, otitis media, which can be infected fluid (chronic Suppurative otitis media C.S.O.M) or transudation fluid (serous otitis media S.O.M/ middle ear effusion) accumulating in the middle ear [16]. With long standing cases chronic otitis media there is a risk of development of a cholesteatoma, which is skin lined, sac like structure. Cholesteatoma is notorious for erosion and destruction of the bone eventually leading to major complications like brain abscess and meningitis [17].The vast majority of cases of Otitis media have a benign clinical course, Otitis Media can lead totransient and permanent deafness, developmental delays or serious extra-cranial or intracranial complications likebrain abscess and meningitis [18].

Middle ear infections leading to delayed speech and language development, academic performance in schools, psychological development, neurological "soft signs" and cognitive ability has been studied extensively in western population and many papers published at the crux of the pathology [19]. But no such research exists for the Indian especially Konkan children. As such due to difference in demographics of the people it is expected that patter $n$ of presentation of Otits media would be different and more subtle as compared to their western cohorts.

The most common type of acquired hearing loss is conductive hearing loss due chronic middle ear effusion also known as Secretory Otitis media. The age distribution in children described in literature is bi-modal with first peak at around 2.5 years of age, then again at around 4.5 years of age when children enter the school. By one estimate about 30\% of preschool children are affected in winter season [20]. The American Academy of Paediatrics estimates an incidence of over 5 million middle ear infections in American children, leading to about 30 million visits to clinics per year. Over 10 million annual antibiotic prescriptions are handed out for treatment of Otitis media. This is disease burden of middle ear effusion [21].

Acoustic Reflex Testing- Acoustic reflex detects the increase in the impedance of the middle ear in response to contraction of the Stapedius muscle within $10 \mathrm{~ms}$, brought about in response to a loud sound of more than usually $80 \mathrm{~dB}$ or higher [22]. The afferent limb of this reflex is the Cochlea and Vestibulo-cochlear nerve, the reflex centre is located in the brain stem and efferent limb is via the facial nerve. The vertical segment of facial nerve supplies the Stapedius muscle; the nerve to Stapedius branch is proximal to the geniculate ganglion of Facial nerve. Same ear and opposite ear can be stimulated with sound and impedance increase recorded this is called uncrossed (ipsilateral) acousticreflex andcrossed (contralateral) acousticreflex test respectively [23].

This reflex becomes absent in presence of slightest degree of hearing loss, hence by itself is a sensitive test for detection of deafness. Same sided or uncrossed (ipsilateral) acoustic reflex test is easy to perform and can be done by the same probe which is used to record Tympanometry. This test was done and reflex was studied. If Stapedius muscle contraction within a time span of $10 \mathrm{~ms}$ was elicited this test was designated as "pass" and formed another criteria for screening for Conductive hearing loss and middle ear disease [24].

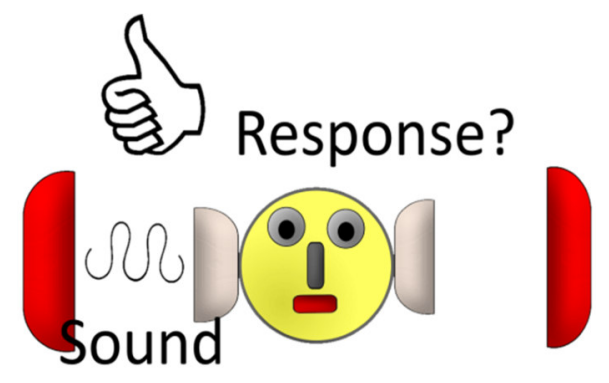

Fig-2: Uncrossed (ipsilateral) Acoustic Reflex Testing (drawn by first author) [24] 
Jerger's classification for Tympanometric configurations [25]- A type "A" tympanogram is normal middle function and conveys ease of $226 \mathrm{~Hz}$ energy flow across the middle ear when the ear canal pressure is at zero pressure $(0$ daPa), with reduced energy flow if pressure is increased or decreased. This creates the classical triangular shape of a Type "A" Impedance audiogram. This indicates optimal healthy middle ear function [26].

A type "B" tympanogram suggests that there is little movement of the middle ear with pressure variation, which can be due to Otitis media with effusion, tympanic membrane atelectasis, or a tympanic membrane perforation. A type "C" tympanogram indicates that the middle ear is under negative pressure, shifting the whole curve to right [27].

Utility of this Dervan Middle ear effusion Screening (test) protocol- The quality of a test or a protocol is dependent on the following Biostatistical parameters namely validity, reliability, sensitivity, specificity and predictive values of positive/ negative tests. The validity of a screening test must be upheld by comparison with a "Gold standard" test which in our study is direct observation of the Middle ear effusion by two independent surgeons.

Since the test subjects were selected at random from children who attended the paediatric clinic there is no reason to assume external validity of the protocol especially on Konkan population for which this was developed. Reproducibility or reliability is open to further studies at our department or other hospitals which will validate this parameter.

The sensitivity of a protocol is its ability to accurately screen population who do have trait. The sensitivity of a protocol is its ability to accurately screen out or eliminate population who do not have trait or disease risk [28].

Table-4: Evaluation of the sensitivity and specificity of the Protocol against "Gold standard" Endoscopy/ Otoscopic examination of the ear

\begin{tabular}{|c|c|c|}
\hline \multirow{2}{*}{ Tympanometry } & \multicolumn{2}{|c|}{ Dervan child Middle ear effusion protocol } \\
\hline & \multicolumn{2}{|c|}{ Endoscopic defined effusion } \\
\cline { 2 - 3 } & Yes & No \\
\hline Positive test (47) & 44 & 49 \\
\hline Negative test (53) & 4 & 52 \\
\hline Total $(\mathrm{n}=100)$ & 48 & \multicolumn{2}{|c|}{5} \\
\hline
\end{tabular}

Dervan child Middle ear effusion protocol is $91.67 \%$ (92\%) sensitive and 94.23 (94\%) specific is detection of Middle ear effusion. The Positive predictive value of the test is 93.6 (94\%) and negative predictive value stands at 92.4 (92\%). Chisquare value, first degree of freedom, at Confidence interval of $95 \%(\mathrm{p}=0.05)$ is found to be $73.93(64.139)$ at $\mathrm{p}=0.05$ is statistically significant. Fischer's exact test has a value of $2.9 \mathrm{E}-17$, Crammer's V value of the test is V=0.84419 which are also very significant.

Contributions by three authors- The senior author and the third author were involved in the supervised audiological testing, collection of data and statistical analysis of the data. The second author being the Paediatric specialist provided technical insights into the clinical framework of the research. First author wrote the manuscript which was discussed and agreed upon by the other authors.

\section{Conclusion}

Middle ear infections leading to delayed speech and language development, academic performance in schools, psychological development, neurological "soft signs" and cognitive ability has been studied extensively in western population and many papers published at the crux of the pathology But no such research exists for the Indian especially Konkan children. As such due to difference in demographics of the people it is expected that patter $n$ of presentation of
Otits media would be different and more subtle as compared to their western cohorts. Dervan child Middle ear effusion protocol can be used in a cost efficient, scalable and sustainable method of screening children for Middle ear effusion. Tympanometry in selected high risk population is an accurate and reliable test for detection of Middle ear effusion. This reproducibility of this protocol has to be validated by further studies in our hospital and elsewhere. 


\section{Original Research Article}

\section{Funding: Nil, Conflict of interest: Nil \\ Permission from IRB: Yes}

Ethical Standards: Ethical committee approval obtained dated 17/1/2018 Official Order Refer: BLKW/ $\mathrm{RMC} / \mathrm{IEC} / 26 / 2018(11)$

\section{References}

1. Davis JM, Elfenbein J, Schum R, Bentler RA. Effects of mild and moderate hearing impairments on language, educational, and psychosocial behavior of children. J Speech Hear Disord. 1986 Feb; 51 (1):53-62. PMID: 3945060 DOI:10.1044/jshd..5101.53

2. American Speech-Language-Hearing Association. Guidelines for Audiologic Screening [Guidelines]. Available from www.asha.org/policy. DOI: 10.1044/ policy. GL1997-00199 ASHA 34:81-87, 1992.

3. Gelfand SA, Silman S. Future perspectives in hearing and aging: clinical and research needs. Semin Hear 1985; 6:207-219.

4. American Speech-Language-Hearing Association. Guidelines for screening for hearing impairments and middle ear disorders. Asha, April 1990, April 32 (Suppl. 2), 17-24.

5. Harford ER, Bess FH, Bluestone CD, Klein JO eds. Impedance Screening for Middle Ear Disease in Children. New York: Grune\& Stratton, 1978.

6. Himelfarb MZ, Popelka GR, Shanon E. Tympanometry in normal neonates. J Speech Hear Res. 1979 Mar;22 (1): 179-91.

7. Paradise JL, Smith CG, Bluestone CD. Tympanometric detection of middle ear effusion in infants and young children. Pediatrics. 1976 Aug; 58 (2): 198-210.

8. Margolis RH, Heller JW. Screening tympanometry: criteria for medical referral. Audiology. 1987;26 (4):197-208.

9. Hunter LL, Margolis RH. Multi frequency Tympanometry: Current Clinical Application. Am J Audiol. 1992 Jul 1;1(3):33-43. doi: 10.1044/1059-0889. 0103.33.

10. Kasliwal N, Joshi S, Pareek SM. Determinants of sensorineural hearing loss in chronic middle-ear disease. Indian J Otolaryngol Head Neck Surg. 2004 Oct; 56(4):269-73. doi: 10.1007/BF02974385.
11. Hammer, Ø.,Harper,D.A.T., Ryan,P.D.2001. PAST: Paleontological statistics software package for education and data analysis. Palaeontologia Electronica 4 (1): 9pp. http://palaeo-electronica.org/ 2001_1/past/ issue1_01.htm.

12. Feinstein AR. Clinical epidemiology the architecture of clinical research, Philadelphia, WB Saunders. 1985;10(1):111-45.

13. Roland PS. Chronic suppurative otitis media: a clinical overview. Ear Nose Throat J. 2002 Aug; 81 (8 Suppl 1): 8-10.

14. Briggs RJ, Luxford WM. Chronic ear surgery: a historical review. Am J Otol. 1994 Jul; 15 (4): 558-67.

15. Bluestone CD, Klein JO. Otitis $\mathrm{m}$ edia, atelectasis, and Eustachian tube dysfunction. In: Bluestone CD, Stool SE, Kenna MA, eds. Pediatric Otolaryngology, 3rded. Philadelphia: WB Saunders, 1996.

16. Pau HW. [Eustachian tube and middle ear mechanics]. HNO. 2011 Oct;59(10):953-63. doi: 10. 1007 /s00106-011-2368-y.

17. PROCTOR B. The development of the middle ear spaces and their surgical significance. J Laryngol Otol. 1964 Jul;78:631-48

18. Jackler RK. The surgical anatomy of cholesteatoma. Otolaryngol Clin North Am. 1989 Oct; 22 (5): 883-96.

19. Shim HJ, Park $\mathrm{CH}$, Kim MG, et al. A pre- and postoperative bacteriological study of chronic suppurative otitis media. Infection. 2010 Dec;38(6): 447-52. doi: 10.1007/s15010-010-0048-6. Epub 2010 Aug 11.

20. Yeo SG, Park DC, Hong SM, et al. Bacteriology of chronic suppurative otitis media--a multicenter study. Acta Otolaryngol. 2007 Oct;127(10):1062-7. DOI:10. 1080/ 00016480601126978

21. Tos M, Poulsen G. Attic retractions following secretory otitis. Acta Otolaryngol. 1980 May-Jun; 89 (5-6): 479-86.

22. Feeney, M. P., \& Sanford, C. A. Detection of the acousticstapedius reflex in infants using wideband energy reflectance and admittance. journal of the American Academy of Audiology, 2005;16(5):278-290. 
23. De Chicchis, A. R., Todd N. W. \& Nozza R. Developmental changes in aural acoustic admittance measurements. journal of the American Academy of Audiology, 2000;11(2), 97-102.

24. Meyer SE, Jardine CA, Deverson W. et al. Developmental changes in tympanometry: a case study. Br J Audiol. 1997 Jun;31(3):189-95.

25. Jerger J. Clinical experience with impedance audiometry. Arch Otolaryngol. 1970 Oct; 92 (4): 311-24.
26. Jerger J, Anthony L, Jerger S, et al. Studies in impedance audiometry. 3. Middle ear disorders. Arch Otolaryngol. 1974 Mar;99(3):165-71.

27. Alaerts J, Luts H, Wouters J. Evaluation of middle ear function in young children: clinical guidelines for the use of $226-$ and $1,000-\mathrm{Hz}$ tympanometry. Otol Neurotol. 2007 Sep;28(6):727-32.

28. Antonella B, Giovanni D. C. Fundamentals of Clinical Research: Bridging Medicine, Statistics and Operations .75(3):1-3. International Statistical Review. Campbell, M. J.2007.ISSN 0306-7734. 2007. DOI:10. 1111/j. 1751-5823.2007.00030_3.x

How to cite this article?

Rajamani S.K, Choudhary V.C, Mogre D.A. Tympanometric screening for Otitis media of paediatric patients with respiratory tract infection in rural setting a prospective observational study. Trop J Ophthalmol Otolaryngol.2019;4(2): 100-108. doi: 10.17511/jooo.2019.i02.06 